\title{
Some common fixed point results for weakly compatible mappings in cone metric type space
}

\author{
Hamidreza Rahimi, Pasquale Vetro, and Ghasem \\ Soleimani Rad
}




\title{
SOME COMMON FIXED POINT RESULTS FOR WEAKLY COMPATIBLE MAPPINGS IN CONE METRIC TYPE SPACE
}

\author{
HAMIDREZA RAHIMI, PASQUALE VETRO, AND GHASEM SOLEIMANI RAD
}

Received 18 September, 2012

\begin{abstract}
In this paper we consider cone metric type spaces which are introduced as a generalization of symmetric and metric spaces by Khamsi and Hussain in 2010. Then we prove several common fixed point for weakly compatible mappings in cone metric type spaces. All results are proved in the settings of a solid cone, without the assumption of continuity of the mappings.
\end{abstract}

2000 Mathematics Subject Classification: 47H10; 54H25; 47H09

Keywords: cone metric type space, common fixed point, coincidence point, weakly compatible mappings, solid cone

\section{INTRODUCTION}

In 1922, Banach proved the famous contraction mapping principle [4]. Afterward, other authors considered various definitions of contractive mappings and proved several fixed and common fixed point theorems [6, 13, 19, 25]. In 1976, Jungck [18] proved a common fixed point theorem for two commuting mappings. This theorem has many applications but it requires the continuity of one of the two mappings. In 1996, Jungck [16] defined a pair of self-mappings to be weakly compatible if they commute at their coincidence points. In the sequel, Jungck and Rhoades [17] proved some fixed and common fixed point theorems for noncommuting and compatible mappings in metric spaces.

In 2007, Huang and Zhang [14] introduced cone metric spaces and proved some fixed point theorems. Several fixed and common fixed point results in cone metric spaces are proved in [1,5,9-12,21,22, 24, 26,27].

In 1931, Wilson [28] introduced symmetric spaces, as metric-like spaces lacking the triangle inequality. Recently, Khamsi and Hussain $[20,21]$ defined a new type of spaces which they called cone metric type spaces. Afterward, other authors proved fixed point theorems in metric type space and cone metric type spaces [7, 15,23]. The purpose of this paper is to generalize and unify the common fixed point theorems for weakly compatible mappings of Abbas and Jungck [1], Abbas and Rhoades [2], Arshad et al. [3], Huang and Zhang [14], on cone metric type spaces. 


\section{Preliminaries}

We recall some definitions and results that we will use in the sequel. Throughout this article we denote by $\mathbb{R}$ the set of all real numbers and by $\mathbb{N}$ the set of positive integers.

Definition 1 (See [28]). Let $X$ be a nonempty set. Suppose that the mapping $D: X \times X \rightarrow[0,+\infty)$ satisfies

(S1) $D(x, y)=0 \Longleftrightarrow x=y$;

(S2) $D(x, y)=D(y, x)$,

for all $x, y \in X$. Then $D$ is called a symmetric on $X$ and $(X, D)$ is called a symmetric space.

Definition 2 (See $[8,14])$. Let $\mathrm{E}$ be a real Banach space and $P$ a subset of $E$. Then $P$ is called a cone if and only if

(a) $P$ is closed, non-empty and $P \neq\{0\}$;

(b) $a, b \in \mathbb{R}, a, b \geq 0$ and $x, y \in P$ imply that $a x+b y \in P$;

(c) if $x \in P$ and $-x \in P$, then $x=0$.

Given a cone $P \subset E$, we define a partial ordering $\leq$ with respect to $P$ by

$$
x \leq y \Longleftrightarrow y-x \in P .
$$

We shall write $x<y$ if $x \leq y$ and $x \neq y$. Also, we write $x \ll y$ if and only if $y-x \in \operatorname{int} P$ (where int $P$ is the interior of $P$ ). If int $P \neq \varnothing$, the cone $P$ is called solid. The cone $P$ is called normal if there is a number $k>0$ such that for all $x, y \in E$,

$$
0 \leq x \leq y \Longrightarrow\|x\| \leq k\|y\| .
$$

The least positive number satisfying the above condition is called the normal constant of $P$. In the sequel we always suppose that $E$ is a real Banach space, $P$ is a solid cone in $E$, and $\leq$ is a partial ordering with respect to $P$.

Example 1. (See [24])

(i) Let $E=C_{\mathbb{R}}[0,1]$ with the supremum norm and $P=\{f \in E: f \geq 0\}$. Then, $P$ is a normal cone with normal constant $k=1$.

(ii) Let $E=C_{\mathbb{R}}^{1}[0,1]$ with the norm $\|f\|=\|f\|_{\infty}+\left\|f^{\prime}\right\|_{\infty}$ and consider the cone $P=\{f \in E: f \geq 0\}$. Then $P$ is a non-normal cone.

Definition 3 (See [14]). Let $X$ be a nonempty set. Suppose that the mapping $d: X \times X \rightarrow E$ satisfies

(d1) $0 \leq d(x, y)$ for all $x, y \in X$ and $d(x, y)=0$ if and only if $x=y$;

(d2) $d(x, y)=d(y, x)$ for all $x, y \in X$

(d3) $d(x, z) \leq d(x, y)+d(y, z)$ for all $x, y, z \in X$.

Then, $d$ is called a cone metric on $\mathrm{X}$ and $(X, d)$ is called a cone metric space. 
Example 2. (See [14]) Let $E=\mathbb{R}^{2}, P=\{(x, y) \in E \mid x, y \geq 0\} \subset \mathbb{R}^{2}, X=\mathbb{R}$ and $d: X \times X \rightarrow E$ such that $d(x, y)=(|x-y|, \alpha|x-y|)$, where $\alpha \geq 0$ is a constant. Then $(X, d)$ is a cone metric space.

Definition 4 (See [7, 20,21]). Let $X$ be a nonempty set, $K \geq 1$ be a real number and $E$ a real Banach space with cone $P$. Suppose that the mapping $d: X \times X \rightarrow E$ satisfies

(cd1) $d(x, y) \geq 0$ for all $x, y \in X$ and $d(x, y)=0$ if and only if $x=y$;

$(c d 2) d(x, y)=d(y, x)$ for all $x, y \in X$;

$(c d 3) d(x, z) \leq K(d(x, y)+d(y, z))$ for all $x, y, z \in X$.

Then $(X, d, K)$ is called a cone metric type space. Obviously, for $K=1$, a cone metric type space is a cone metric space.

Example 3. (See [7]) Let $B=\left\{e_{i} \mid i=1, \ldots, n\right\}$ be an orthonormal basis of $\mathbb{R}^{n}$ with inner product $(\cdot, \cdot)$ and $p>0$. Define

$$
X_{p}=\left\{\left.[x]\left|x:[0,1] \rightarrow \mathbb{R}^{n}, \int_{0}^{1}\right|\left(x(t), e_{j}\right)\right|^{p} d t \in \mathbb{R}, \quad j=1,2, \ldots, n\right\},
$$

where $[x]$ represents the class of equivalence of $x$ with respect to relation of functions equal almost everywhere. Let $E=\mathbb{R}^{n}$ and

$$
P_{B}=\left\{y \in \mathbb{R}^{n} \mid\left(y, e_{i}\right) \geq 0, \quad i=1,2, \ldots, n\right\}
$$

be a solid cone. Define $d: X_{p} \times X_{p} \rightarrow P_{B} \subset \mathbb{R}^{n}$ by

$$
d(f, g)=\sum_{i=1}^{n} e_{i} \int_{0}^{1}\left|\left((f-g)(t), e_{i}\right)\right|^{p} d t, \quad f, g \in X_{p} .
$$

Then $\left(X_{p}, d, K\right)$ is a cone metric type space with $K=2^{p-1}$.

We define convergence in cone metric type spaces as in the cone metric spaces.

Definition 5 (See [20]). Let $(X, d, K)$ be a cone metric type space, $\left\{x_{n}\right\}$ a sequence in $X$ and $x \in X$.

(i) $\left\{x_{n}\right\}$ converges to $x$ if for every $c \in E$ with $0 \ll c$ there exists $n_{0} \in \mathbb{N}$ such that $d\left(x_{n}, x\right) \ll c$ for all $n>n_{0}$, and we write $\lim _{n \rightarrow+\infty} d\left(x_{n}, x\right)=0$

(ii) $\left\{x_{n}\right\}$ is called a Cauchy sequence if for every $c \in E$ with $0 \ll c$ there exists $n_{0} \in$ $\mathbb{N}$ such that $d\left(x_{n}, x_{m}\right) \ll c$ for all $m, n>n_{0}$, and we write $\lim _{n, m \rightarrow+\infty} d\left(x_{n}, x_{m}\right)=$ 0 .

Lemma 1 (See [7]). Let $(X, d, K)$ be a cone metric type space over-ordered real Banach space E. Then the following properties are often used, particularly in dealing with cone metric type spaces in which the cone need not be normal.

$\left(P_{1}\right)$ If $u \leq v$ and $v \ll w$, then $u \ll w$.

$\left(P_{2}\right)$ If $0 \leq u \ll c$ for each $c \in \operatorname{int} P$, then $u=0$.

$\left(P_{3}\right)$ If $u \leq \lambda u$ where $u \in P$ and $0 \leq \lambda<1$, then $u=0$. 
$\left(P_{4}\right)$ Let $x_{n} \rightarrow 0$ in $E$ and $0 \ll c$. Then there exists a positive integer $n_{0}$ such that $x_{n} \ll c$ for each $n>n_{0}$.

Definition 6 (See [17]). Let $f$ and $g$ be two self-mappings defined on a set $X$. If $f w=g w=z$ for some $z \in X$, then $w$ is called a coincidence point of $f$ and $g$, and $z$ is called a point of coincidence of $f$ and $g$. The mappings $f$ and $g$ are said to be weakly compatible if they commute at every coincidence point, that is, if $f g w=g f w$ for all coincidence points $w$.

Lemma 2 (See [1]). Let $f$ and $g$ be weakly compatible self-mappings on a set $X$. If $f$ and $g$ have a unique point of coincidence $z=f w=g w$, then $z$ is the unique common fixed point of $f$ and $g$.

\section{MAIN RESULTS}

The following theorem, that extends and improves Theorem 2 of [3] and Corollary 2.10 of [2] in a cone metric type space, is our main result.

Theorem 1. Let $(X, d, K)$ be a cone metric type space with constant $K \geq 1$ and $P$ a solid cone. Suppose that $f, g$ and $T$ are three self-mappings on $X$, satisfying $f(X) \cup g(X) \subset T(X)$, and

$$
\begin{aligned}
d(f x, g y) \leq & p(x, y) d(T x, T y)+q(x, y) d(f x, T x) \\
& +r(x, y) d(g y, T y)+2 t(x, y)\left[\frac{d(f x, T y)+d(g y, T x)}{2}\right]
\end{aligned}
$$

for all $x, y \in X$, where $p, q, r, t: X \times X \rightarrow\left[0, \frac{1}{K}\right)$ are real functions such that

$$
\sup _{x, y \in X}\left\{K p(x, y)+(K+1) \max \{q(x, y), r(x, y)\}+\left(K^{2}+K\right) t(x, y)\right\} \leq \lambda<1 .
$$

If one of $f(X), g(X)$ or $T(X)$ is a complete subspace of $X$, then $\{f, T\}$ and $\{g, T\}$ have a unique point of coincidence in $X$. Moreover if $\{f, T\}$ and $\{g, T\}$ are weakly compatible, then $f, g$ and $T$ have a unique common fixed point.

Proof. Suppose $x_{0}$ is an arbitrary point of $X$. Since $f(X) \subset T(X)$, there exists $x_{1} \in X$ such that $f x_{0}=T x_{1}=y_{1}$. Since $g(X) \subset T(X)$, there exists $x_{2} \in X$ such that $g x_{1}=T x_{2}=y_{2}$. If we continue in this manner, then

$$
\begin{array}{lll}
\exists x_{2 n+1} \in X & \text { such that } & y_{2 n+1}=f x_{2 n}=T x_{2 n+1} \\
\exists x_{2 n+2} \in X & \text { such that } & y_{2 n+2}=g x_{2 n+1}=T x_{2 n+2},
\end{array}
$$

for $n=0,1, \ldots$.

In the sequel we denote $p(x, y), q(x, y), r(x, y), t(x, y)$ respectively with $p, q, r, t$. Now, we have

$$
\begin{aligned}
d\left(y_{2 n+1}, y_{2 n+2}\right) & =d\left(f x_{2 n}, g x_{2 n+1}\right) \\
& \leq p d\left(T x_{2 n}, T x_{2 n+1}\right)+q d\left(f x_{2 n}, T x_{2 n}\right)
\end{aligned}
$$




$$
\begin{aligned}
& +r d\left(g x_{2 n+1}, T x_{2 n+1}\right) \\
& +t\left[d\left(f x_{2 n}, T x_{2 n+1}\right)+d\left(g x_{2 n+1}, T x_{2 n}\right)\right] \\
& =p d\left(y_{2 n}, y_{2 n+1}\right)+q d\left(y_{2 n+1}, y_{2 n}\right)+r d\left(y_{2 n+2}, y_{2 n+1}\right) \\
& +t\left[d\left(y_{2 n+1}, y_{2 n+1}\right)+d\left(y_{2 n+2}, y_{2 n}\right)\right] \\
& \leq p d\left(y_{2 n}, y_{2 n+1}\right)+q d\left(y_{2 n+1}, y_{2 n}\right)+r d\left(y_{2 n+2}, y_{2 n+1}\right) \\
& +t K\left[d\left(y_{2 n+2}, y_{2 n+1}\right)+d\left(y_{2 n+1}, y_{2 n}\right)\right] \\
& =(p+q+t K) d\left(y_{2 n}, y_{2 n+1}\right)+(r+t K) d\left(y_{2 n+1}, y_{2 n+2}\right),
\end{aligned}
$$

which implies that

$$
d\left(y_{2 n+1}, y_{2 n+2}\right) \leq \frac{p+q+t K}{1-r-t K} d\left(y_{2 n}, y_{2 n+1}\right) .
$$

Similarly,

$$
d\left(y_{2 n+3}, y_{2 n+2}\right) \leq \frac{p+r+t K}{1-q-t K} d\left(y_{2 n+2}, y_{2 n+1}\right) .
$$

Since $\lambda<1$, from

$$
K p(x, y)+K q(x, y)+\lambda r(x, y)+K^{2} t(x, y)+\lambda K t(x, y) \leq \lambda
$$

and

$$
K p(x, y)+\lambda q(x, y)+K r(x, y)+K^{2} t(x, y)+\lambda K t(x, y) \leq \lambda,
$$

that holds by relation (3.2), we have

$$
K \frac{p(x, y)+q(x, y)+K t(x, y)}{1-r(x, y)-K t(x, y)} \leq \lambda
$$

and

$$
K \frac{p(x, y)+r(x, y)+K t(x, y)}{1-q(x, y)-K t(x, y)} \leq \lambda
$$

holds for all $x, y \in X$. Therefore

$$
d\left(y_{n}, y_{n+1}\right) \leq \frac{\lambda}{K} d\left(y_{n-1}, y_{n}\right) \quad \text { for all } n \in \mathbb{N}
$$

and hence

$$
d\left(y_{n}, y_{n+1}\right) \leq \frac{\lambda}{K} d\left(y_{n-1}, y_{n}\right) \leq \cdots \leq\left(\frac{\lambda}{K}\right)^{n} d\left(y_{0}, y_{1}\right) .
$$

Now, for $m>n$ we have

$$
\begin{aligned}
d\left(y_{n}, y_{m}\right) & \leq K d\left(y_{n}, y_{n+1}\right)+K^{2} d\left(y_{n+1}, y_{n+2}\right)+\cdots+K^{m-n} d\left(y_{m-1}, y_{m}\right) \\
& \leq \frac{1}{K^{n-1}}\left(\lambda^{n}+\lambda^{n+1}+\cdots+\lambda^{m-1}\right) d\left(y_{0}, y_{1}\right) \\
& \leq \frac{1}{K^{n-1}} \frac{\lambda^{n}}{1-\lambda} d\left(y_{0}, y_{1}\right) \rightarrow 0 \quad \text { in } E \text { as } \quad n \rightarrow+\infty .
\end{aligned}
$$


Now, by $\left(P_{1}\right)$ and $\left(P_{4}\right)$, it follows that for every $c \in \operatorname{int} P$ there exists a positive integer $n_{0}$ such that $d\left(y_{n}, y_{m}\right) \ll c$ for every $m>n>n_{0}$, so $\left\{y_{n}\right\}$ is a Cauchy sequence. Suppose that $T(X)$ is a complete subspace of $X$, then $\left\{y_{n}\right\}$ is convergent in $T(X)$ and there exists $v \in X$ such that $\lim _{n \rightarrow+\infty} T x_{2 n}=\lim _{n \rightarrow+\infty} y_{2 n}=v$. Since $T$ is a self-mapping, there exists $u \in X$ such that $T u=v$. Now, we prove that $f u=v$. By (3.1), we obtain

$$
\begin{aligned}
d(f u, v) & \leq K\left[d\left(f u, g x_{2 n+1}\right)+d\left(g x_{2 n+1}, v\right)\right] \\
& \leq K\left[p d\left(T u, T x_{2 n+1}\right)+q d(f u, T u)+r d\left(g x_{2 n+1}, T x_{2 n+1}\right)\right. \\
& \left.+t\left[d\left(f u, T x_{2 n+1}\right)+d\left(g x_{2 n+1}, T u\right)\right]\right]+K d\left(g x_{2 n+1}, v\right) \\
& =K\left[p d\left(v, y_{2 n+1}\right)+q d(f u, v)+r d\left(y_{2 n+2}, y_{2 n+1}\right)\right. \\
& \left.+t\left[d\left(f u, y_{2 n+1}\right)+d\left(y_{2 n+2}, v\right)\right]\right]+K d\left(y_{2 n+2}, v\right) \\
& \leq p K d\left(v, y_{2 n+1}\right)+q K d(f u, v)+r K d\left(y_{2 n+2}, y_{2 n+1}\right) \\
& +t K^{2}\left[d(f u, v)+d\left(v, y_{2 n+1}\right)\right]+t K d\left(y_{2 n+2}, v\right)+K d\left(y_{2 n+2}, v\right) \\
& =\left(p K+t K^{2}\right) d\left(v, y_{2 n+1}\right)+r K d\left(y_{2 n+2}, y_{2 n+1}\right)+ \\
& K(t+1) d\left(y_{2 n+2}, v\right)+\left(q K+t K^{2}\right) d(f u, v),
\end{aligned}
$$

which implies

$$
\begin{aligned}
\left(1-q K-t K^{2}\right) d(f u, v) & \leq\left(p K+t K^{2}\right) d\left(v, y_{2 n+1}\right)+r K d\left(y_{2 n+2}, y_{2 n+1}\right) \\
& +K(t+1) d\left(y_{2 n+2}, v\right) .
\end{aligned}
$$

Now, using (3.2), we have that

$$
(1-\lambda) d(f u, v) \leq \lambda d\left(v, y_{2 n+1}\right)+\lambda d\left(y_{2 n+2}, y_{2 n+1}\right)+(\lambda+K) d\left(y_{2 n+2}, v\right)
$$

holds for all $n \in \mathbb{N}$. Since $\left\{y_{n}\right\}$ converges to $v$ and $d\left(y_{2 n+2}, y_{2 n+1}\right) \rightarrow 0$ in $E$ as $n \rightarrow+\infty$, for every $c \in \operatorname{int} P$ there exists $n_{0} \in \mathbb{N}$ such that for any $n>n_{0}$, we have

$$
\begin{aligned}
d\left(v, y_{2 n+1}\right) & \ll \frac{(1-\lambda) c}{3 \lambda}, \\
d\left(y_{2 n+2}, y_{2 n+1}\right) & \ll \frac{(1-\lambda) c}{3 \lambda}, \\
d\left(y_{2 n+2}, v\right) & \ll \frac{(1-\lambda) c}{3(\lambda+K)} .
\end{aligned}
$$

It follows that $d(f u, v) \ll c$ for every $c \in \operatorname{int} P$, and by $\left(P_{2}\right)$ we have $d(f u, v)=0$, that is, $f u=v$. So, we have $f u=T u=v$, that is, $v$ is a point of coincidence of the mappings $f$ and $T$, and $u$ is a coincidence point of the mapping $f$ and $T$.

Similarly, by (3.1) and (3.2) we conclude $d(v, g u) \ll c$ for every $c \in \operatorname{int} P$, and we have $d(g u, v)=0$ by $\left(P_{2}\right)$, that is, $g u=v$. So, we have $g u=T u=v$, that is, $v$ is a point of coincidence of the mappings $g$ and $T$, and $u$ is a coincidence point of the mappings $g$ and $T$. Hence $f u=g u=T u=v$. 
Now we shall show that $v$ is the unique point of coincidence of the pairs $\{f, T\}$ and $\{g, T\}$. Let $v^{\prime}$ be also a point of coincidence of these three mappings, then $f u^{\prime}=g u^{\prime}=T u^{\prime}=v^{\prime}$ for $u^{\prime} \in X$. From (3.1), we have

$$
\begin{aligned}
d\left(v, v^{\prime}\right) & =d\left(f u, g u^{\prime}\right) \\
& \leq p d\left(T u, T u^{\prime}\right)+q d(f u, T u)+r d\left(g u^{\prime}, T u^{\prime}\right) \\
& +t\left[d\left(f u, T u^{\prime}\right)+d\left(g u^{\prime}, T u\right)\right] \\
& =p d\left(v, v^{\prime}\right)+q d(v, v)+r d\left(v^{\prime}, v^{\prime}\right)+t\left[d\left(v, v^{\prime}\right)+d\left(v^{\prime}, v\right)\right] \\
& =(p+2 t) d\left(v, v^{\prime}\right) \\
& \leq \lambda d\left(v, v^{\prime}\right),
\end{aligned}
$$

and (by $\left(P_{3}\right)$ ) it follows that $v=v^{\prime}$. If the pairs $\{f, T\}$ and $\{g, T\}$ are weakly compatible, then $v$ is the unique common fixed point of $f, g$ and $T$ by Lemma (2).

The following result is a consequence of Theorem 1.

Theorem 2. Let $(X, d, K)$ be a cone metric type space and $P$ a solid cone. Suppose that $f, g$ and $T$ are three self-mappings on $X$, satisfying $f(X) \cup g(X) \subset T(X)$, and

$$
d(f x, g y) \leq \alpha M_{x, y}(f, g, T),
$$

where $\alpha \in\left(0, \min \left\{\frac{1}{K+1}, \frac{2}{K(K+1)}\right\}\right)$ for $K \geq 1$ and

$$
M_{x, y}(f, g, T) \in\left\{d(T x, T y), d(f x, T x), d(g y, T y), \frac{d(f x, T y)+d(g y, T x)}{2}\right\},
$$

for all $x, y \in X$. If one of $f(X), g(X)$ or $T(X)$ is a complete subspace of $X$, then $\{f, T\}$ and $\{g, T\}$ have a unique point of coincidence in $X$. Moreover if $\{f, T\}$ and $\{g, T\}$ are weakly compatible, then $f, g$ and $T$ have a unique common fixed point.

Proof. First, we consider the following subsets of $X \times X$ :

$$
\begin{aligned}
& \mathrm{A}=\{(x, y) \in X \times X: d(f x, g y) \leq d(T x, T y)\} ; \\
& \mathrm{B}=\{(x, y) \in X \times X \backslash A: d(f x, g y) \leq d(f x, T x)\} ; \\
& \mathrm{C}=\{(x, y) \in X \times X \backslash(A \cup B): d(f x, g y) \leq d(g y, T y)\},
\end{aligned}
$$

and we define the functions $p, q, r, t: X \times X \rightarrow[0,1 / K)$ as following

$$
\begin{gathered}
p(x, y)=\left\{\begin{array}{ll}
\alpha, & \text { if } \quad(x, y) \in A \\
0, & \text { otherwise }
\end{array} \quad, \quad q(x, y)=\left\{\begin{array}{ll}
\alpha, & \text { if }(x, y) \in B \\
0, & \text { otherwise }
\end{array},\right.\right. \\
r(x, y)=\left\{\begin{array}{ll}
\alpha, & \text { if }(x, y) \in C \\
0, & \text { otherwise }
\end{array}, t(x, y)= \begin{cases}\alpha / 2, & \text { if }(x, y) \in(A \cup B \cup C)^{c} \\
0, & \text { otherwise }\end{cases} \right.
\end{gathered}
$$

Now, the mappings $f, g$ and $T$ satisfy the conditions (3.1) and (3.2) with respect to the previous functions if $\alpha<\min \left\{\frac{1}{K+1}, \frac{2}{K(K+1)}\right\}$, and so Theorem 2 follows by Theorem 1. 
Remark 1. Proceeding as in the proof of Theorem 1, we can prove that Theorem 2 holds for $\alpha<\frac{2}{K(K+1)}$ also when $1 \leq K \leq 2$. So Theorem 2 extends Corollary 2.5 of [2] in the setting of a cone metric type space.

The following results is obtained from Theorem 1.

Corollary 1. Let $(X, d, K)$ be a cone metric type space with constant $K \geq 1$ and $P$ a solid cone. Suppose that the mappings $f, g$ and $T$ are three self-maps on $X$, satisfying

$f(X) \cup g(X) \subset T(X)$, and

$d(f x, g y) \leq p d(T x, T y)+q d(f x, T x)+r d(g y, T y)+t[d(f x, T y)+d(g y, T x)]$,

for all $x, y \in X$, where $p, q, r, t \in\left[0, \frac{1}{K}\right)$ and

$$
K p+(K+1) \max \{q, r\}+\left(K^{2}+K\right) t<1 .
$$

If one of $f(X), g(X)$ or $T(X)$ is a complete subspace of $X$, then $\{f, T\}$ and $\{g, T\}$ have a unique point of coincidence in $X$. Moreover if $\{f, T\}$ and $\{g, T\}$ are weakly compatible, then $f, g$ and $T$ have a unique common fixed point.

Corollary 2. Let $(X, d, K)$ be a cone metric type space with constant $K \geq 1$ and $P$ a solid cone. Suppose that $f$ and $T$ are two self-mappings on $X$, satisfying $f(X) \subset$ $T(X)$ and

$d(f x, f y) \leq p d(T x, T y)+q d(f x, T x)+r d(f y, T y)+t[d(f x, T y)+d(f y, T x)]$,

for all $x, y \in X$, where $p, q, r, t \in\left[0, \frac{1}{K}\right)$ and

$$
K p+(K+1) \max \{q, r\}+\left(K^{2}+K\right) t<1 .
$$

If one of $f(X)$ or $T(X)$ is a complete subspace of $X$, then $\{f, T\}$ has a unique point of coincidence in $X$. Moreover if $\{f, T\}$ is weakly compatible, then $f$ and $T$ have a unique common fixed point.

Proof. In (3.5), set $f=g$. It follows from Corollary 1 that $\{f, T\}$ have a unique common fixed point $v$.

Corollary 3. Let $(X, d, K)$ be a cone metric type space with constant $K \geq 1$ and $P$ a solid cone. Suppose that $f$ and $T$ are two self-mappings on $X$, satisfying $f(X) \subset$ $T(X)$ and

$$
\begin{aligned}
d(f x, f y) & \leq a_{1} d(T x, T y)+a_{2} d(f x, T x)+a_{3} d(f y, T y) \\
& +a_{4} d(f x, T y)+a_{5} d(f y, T x)
\end{aligned}
$$

for all $x, y \in X$, where $a_{i} \geq 0$ for $i=1,2, \ldots, 5$ and

$$
K a_{1}+(K+1) \max \left\{a_{2}, a_{3}\right\}+\left(K^{2}+K\right) a_{4}<1,
$$




$$
K a_{1}+(K+1) \max \left\{a_{2}, a_{3}\right\}+\left(K^{2}+K\right) a_{5}<1 .
$$

If one of $f(X)$ or $T(X)$ is a complete subspace of $X$, then $\{f, T\}$ has a unique point of coincidence in $X$. Moreover if $\{f, T\}$ is weakly compatible, then $f$ and $T$ have a unique common fixed point.

\section{Proof. See [7].}

Remark 2. Corollaries 2.11 and 2.12 in [2] can be generalized into cone metric type space by Corollary 2. Also, some results in [7] can be obtained by our corollaries.

The following corollary is obtained from Theorem 2.

Corollary 4. Let $(X, d, K)$ be a cone metric type space and $P$ a solid cone. Suppose that $f$ and $T$ are two self-mappings on $X$, satisfying $f(X) \subset T(X)$, and

$$
d(f x, f y) \leq \alpha M_{x, y}(f, T),
$$

where $\alpha \in\left(0, \min \left\{\frac{1}{K+1}, \frac{2}{K(K+1)}\right\}\right)$ for $K \geq 1$ and

$$
M_{x, y}(f, T) \in\left\{d(T x, T y), d(f x, T x), d(f y, T y), \frac{d(f x, T y)+d(f y, T x)}{2}\right\},
$$

for all $x, y \in X$. If one of $f(X)$ or $T(X)$ is a complete subspace of $X$, then $\{f, T\}$ have a unique point of coincidence in $X$. Moreover if $\{f, T\}$ is weakly compatible, then $f$ and $T$ have a unique common fixed point.

Proof. In Theorem 2, set $f=g$.

Example 4. Let $X=E=\mathbb{R}$ and $P=[0,+\infty)$. Suppose that $d(x, y)=|x-y|^{2}$ for all $x, y \in X$. Then $(X, d, K)$ is a cone metric type space with $K=2$ by Minkowski inequality. Let $f, T: X \rightarrow X$ be two mappings defined as follows

$$
f x=\frac{1}{\sqrt{4}}(2 x+3) \quad \text { and } \quad T x=2 x+3,
$$

where $x \in X$. Since $f(X)=T(X)=X$, we have $f(X) \subset T(X)$. Also,

$$
d(f x, f y)=\left|\frac{1}{\sqrt{4}}(2 x+3)-\frac{1}{\sqrt{4}}(2 y+3)\right|^{2}=\frac{1}{4} d(T x, T y)
$$

and so (3.11) holds with $\alpha=\frac{1}{4}$. According to Corollary (4), $\{f, T\}$ have a unique point of coincidence in $X$. Indeed $v=0$ is the unique point of coincidence of $\{f, T\}$ and $u=\frac{-3}{2}$ is a coincidence point of the mappings $f$ and $T$. 


\section{ACKNOWLEDGEMENTS}

The first and the third authors were supported by Central Tehran Branch of IAU and the second author by Università degli Studi di Palermo. Also, the authors thank the anonymous referee for his/her valuable suggestions that helped to improve the final version of this paper.

\section{REFERENCES}

[1] M. Abbas and G. Jungck, "Common fixed point results for noncommuting mappings without continuity in cone metric spaces," J. Math. Anal. Appl., vol. 341, no. 1, pp. 416-420, 2008.

[2] M. Abbas, B. E. Rhoades, and T. Nazir, "Common fixed points for four maps in cone metric spaces," Appl. Math. Comput., vol. 216, no. 1, pp. 80-86, 2010.

[3] M. Arshad, A. Azam, and P. Vetro, "Some common fixed point results in cone metric spaces," Fixed Point Theory Appl., vol. 2009, p. 11, 2009.

[4] S. Banach, "Sur les opérations dans les ensembles abstraits et leur application aux équations intégrales," Fundamenta math., vol. 3, pp. 133-181, 1922.

[5] B. S. Choudhury and N. Metiya, "The point of coincidence and common fixed point for a pair of mappings in cone metric spaces," Comput. Math. Appl., vol. 60, no. 6, pp. 1686-1695, 2010.

[6] L. B. Ćirić, "A generalization of Banach's contraction principle," Proc. Am. Math. Soc., vol. 45, pp. 267-273, 1974.

[7] A. S. Cvetković, M. P. Stanić, S. Dimitrijević, and S. Simić, "Common fixed point theorems for four mappings on cone metric type space," Fixed Point Theory Appl., vol. 2011, p. 15, 2011.

[8] K. Deimling, Nonlinear functional analysis. Berlin: Springer-Verlag, 1985.

[9] C. Di Bari and P. Vetro, "Common fixed points in cone metric spaces for $M K$-pairs and $L$-pairs," Ars Comb., vol. 99, pp. 429-437, 2011.

[10] C. Di Bari, R. Saadati, and P. Vetro, "Common fixed points in cone metric spaces for $C J M$-pairs," Math. Comput. Modelling, vol. 54, no. 9-10, pp. 2348-2354, 2011.

[11] C. Di Bari and P. Vetro, " $\varphi$-pairs and common fixed points in cone metric spaces," Rend. Circ. Mat. Palermo (2), vol. 57, no. 2, pp. 279-285, 2008.

[12] C. Di Bari and P. Vetro, "Weakly $\varphi$-pairs and common fixed points in cone metric spaces," Rend. Circ. Mat. Palermo (2), vol. 58, no. 1, pp. 125-132, 2009.

[13] B. Fisher, "Four mappings with a common fixed point," J. Univ. Kuwait, Sci., vol. 8, pp. 131-138, 1981.

[14] L.-G. Huang and X. Zhang, "Cone metric spaces and fixed point theorems of contractive mappings," J. Math. Anal. Appl., vol. 332, no. 2, pp. 1468-1476, 2007.

[15] M. Jovanović, Z. Kadelburg, and S. Radenović, "Common fixed point results in metric-type spaces," Fixed Point Theory Appl., vol. 2010, p. 15, 2010.

[16] G. Jungck, "Common fixed points for noncontinuous nonself maps on nonmetric spaces," Far East J. Math. Sci., vol. 4, no. 2, pp. 199-215, 1996.

[17] G. Jungck and B. E. Rhoades, "Fixed point theorems for occasionally weakly compatible mappings," Fixed Point Theory, vol. 7, no. 2, pp. 287-296, 2006.

[18] G. Jungck, "Commuting mappings and fixed points," Am. Math. Mon., vol. 83, pp. 261-263, 1976.

[19] R. Kannan, "Some results on fixed points," Bull. Calcutta Math. Soc., vol. 60, pp. 71-76, 1968.

[20] M. A. Khamsi and N. Hussain, "KKM mappings in metric type spaces," Nonlinear Anal., Theory Methods Appl., Ser. A, Theory Methods, vol. 73, no. 9, pp. 3123-3129, 2010.

[21] M. A. Khamsi, "Remarks on cone metric spaces and fixed point theorems of contractive mappings," Fixed Point Theory Appl., vol. 2010, p. 7, 2010. 
[22] S. Radojević, L. Paunović, and S. Radenović, "Abstract metric spaces and Hardy-Rogers-type theorems," Appl. Math. Lett., vol. 24, no. 4, pp. 553-558, 2011.

[23] H. Rahimi and G. S. Rad, "Some fixed point results in metric type space," J. Basic Appl. Sci. Res., vol. 2, no. 9, pp. 9301-9308, 2012.

[24] S. Rezapour and R. Hamlbarani, "Some notes on the paper "Cone metric spaces and fixed point theorems of contractive mappings"," J. Math. Anal. Appl., vol. 345, no. 2, pp. 719-724, 2008.

[25] B. E. Rhoades, "A comparison of various definitions of contractive mappings," Trans. Am. Math. Soc., vol. 226, pp. 257-290, 1977.

[26] W. Sintunavarat, Y. J. Cho, and P. Kumam, "Common fixed point theorems for $c$-distance in ordered cone metric spaces," Comput. Math. Appl., vol. 62, no. 4, pp. 1969-1978, 2011.

[27] P. Vetro, "Common fixed points in cone metric spaces," Rend. Circ. Mat. Palermo (2), vol. 56, no. 3, pp. 464-468, 2007.

[28] W. A. Wilson, “On semi-metric spaces,” Am. J. Math., vol. 53, pp. 361-373, 1931.

Authors' addresses

\section{Hamidreza Rahimi}

Department of Mathematics, Faculty of Science, Central Tehran Branch, Islamic Azad University,

P. O. Box 13185/768, Tehran, Iran.

E-mail address: rahimieiauctb.ac.ir

\section{Pasquale Vetro}

Dipartimento di Matematica e Informatica, Università degli Studi di Palermo, Via Archirafi, 34/90123, Palermo, Italy.

E-mail address: vetro@math.unipa.it

\section{Ghasem Soleimani Rad}

Department of Mathematics, Faculty of Science, Central Tehran Branch, Islamic Azad University, P. O. Box 13185/768, Tehran, Iran.

E-mail address: gha.soleimani.sci@iauctb.ac.ir, gh.soleimani2008@gmail.com 